\title{
Long-Term Prognosis of Patients With Mildly Dilated Cardiomyopathy
}

\author{
Hiroaki Kitaoka, MD; Yoshihisa Matsumura, MD; Naohito Yamasaki, MD; \\ Fumiaki Kondo, MD; Takashi Furuno, MD; Yoshinori Doi, MD
}

\begin{abstract}
The long-term prognosis of patients with mildly dilated cardiomyopathy (MDCM) was investigated in 21 patients. MDCM was defined as left ventricular ejection fraction $\leq 40 \%$ and left ventricular end-diastolic volume $\leq 120$ $\mathrm{ml} / \mathrm{m}^{2}$ by left ventriculography. During a follow-up period of $6.8 \pm 3.7$ years, there were 9 cardiac events $(5$ heart failure deaths, 2 sudden deaths, and 2 re-hospitalizations for heart failure). Only in the patients without cardiac events was there a significant decrease in left ventricular size (end-diastolic dimension decreased from $58 \pm 6 \mathrm{~mm}$ to $50 \pm 8 \mathrm{~mm}, \mathrm{p}<0.001$ ) and an improvement in systolic function (fractional shortening increased from $17 \pm 5 \%$ to $26 \pm 11 \%, \mathrm{p}=0.007$ ). However, left atrial dilation was observed in the patients with an event (from $39 \pm 5 \mathrm{~mm}$ to $43 \pm 5 \mathrm{~mm}, \mathrm{p}=0.02$ ). Based on proportional hazard analysis, left ventricular end-diastolic pressure and mean pulmonary artery pressure at diagnosis and left atrial dimension at the time of follow-up were significant predictors of poor outcome. A subset of patients with MDCM has impaired hemodynamics at diagnosis, left atrial dilation at follow-up and a poor prognosis, and must be followed carefully even if the left ventricular dilatation is mild. (Circ J 2002; 66: 557-560)
\end{abstract}

Key Words: Dilated cardiomyopathy; Heart failure; Prognosis

$\mathbf{L}$ eft ventricular (LV) dilatation and systolic dysfunction are the hallmarks of idiopathic dilated cardiomyopathy (DCM)! Although the degree of LV dilatation is generally thought to be one of the predictors of poor outcome, ${ }^{2-5}$ several investigators have reported that the prognosis of patients with mild LV dilatation (MDCM) is variable ${ }^{6-8}$ However, the long-term prognosis and changes in the echocardiographic parameters of such patients are unknown and so we aimed to elucidate the long-term prognosis and predictive factors in patients with MDCM.

\section{Methods}

\section{Patients}

A careful history was obtained from all patients, who also underwent physical examination, routine blood tests, chest radiography, standard ECG, exercise stress testing, echocardiography, and right- and left-heart cardiac catheterization with coronary angiography and left ventriculography. DCM was diagnosed based on exclusion of other causes of LV dysfunction, such as acute myocarditis, significant coronary artery stenosis, valvular disease and/or other secondary myocardial diseases. MDCM was defined by (1) a LV ejection fraction (LVEF) $\leq 40 \%$ with a LV end-diastolic volume $\leq 120 \mathrm{ml} / \mathrm{m}^{2}$ on left ventriculography and (2) the absence of a dip-and-plateau right ventricular pressure caused by a restrictive cardiomyopathy? Therefore, of 144 consecutive patients with DCM, 21 (15\%) were diagnosed with MDCM.

(Received October 25, 2001; revised manuscript received February 18, 2002; accepted February 27, 2002)

Cardiology Division, Department of Medicine and Geriatrics, Kochi Medical School, Kochi, Japan

Mailing address: Yoshinori Doi, MD, Department of Medicine and Geriatrics, Kochi Medical School, Oko-cho, Nankoku-shi, Kochi 783-8505, Japan

\section{Cardiac Catheterization and Angiography}

Right- and left-heart cardiac catheterization was performed with fluid-filled catheters and the mean right atrial pressure, $\mathrm{RV}$ end-diastolic pressure, mean pulmonary artery pressure, pulmonary wedge pressure, and LV end-diastolic pressure were recorded. Cardiac output was measured by the thermodilution method. Biplane left ventriculography was performed in the 30-degree right anterior oblique and 60-degree left anterior oblique projections. Selective coronary angiography was performed using the Judkins technique.

\section{Echocardiography}

Echocardiographic studies were performed with a 3.5 MHz transducer (Toshiba 65A or SSH 160A, Toshiba Co, Nasu, Japan). The LV dimensions, LV fractional shortening and left atrial dimensions were measured at baseline and during the follow-up period. Cardiac dimensions were assessed from the M-mode echocardiograms according to the criteria of the American Society of Echocardiography ${ }^{10}$

\section{Follow-up}

Follow-up started from the time of cardiac catheterization and was accomplished using available medical records and telephone interviews with the patients and/or referring physicians. Follow-up echocardiographic studies were performed at outpatient clinics when the patients were in a stable condition. The specified endpoints were heart failure death, sudden death, and re-hospitalization for worsening heart failure.

\section{Statistical Analysis}

Data are presented as the mean \pm SD. Statistical analysis was carried out using Statview V5.0 (SAS Institute, Cary, NC, USA). The paired t-test was used to compare changes in the same group. The unpaired t-test and chi squared tests 
Table 1 Baseline Characteristics of the 21 Patients With MDCM

\begin{tabular}{|c|c|c|c|}
\hline & $\begin{array}{c}\text { Event (+) } \\
(n=9)\end{array}$ & $\begin{array}{c}\text { Event (-) } \\
(n=12)\end{array}$ & $p$ value \\
\hline \multicolumn{4}{|l|}{ Clinical characteristics } \\
\hline Male & $7(78 \%)$ & $9(75 \%)$ & 0.66 \\
\hline Age at diagnosis (years) & $55 \pm 9$ & $62 \pm 9$ & 0.13 \\
\hline NYHA class at diagnosis & $2.6 \pm 1.1$ & $2.1 \pm 0.7$ & 0.18 \\
\hline Atrial fibrillation (\%) & $4(44 \%)$ & $4(33 \%)$ & 0.68 \\
\hline Cardiothoracic ratio (\%) & $53 \pm 4$ & $52 \pm 3$ & 0.27 \\
\hline \multicolumn{4}{|l|}{ Hemodynamic findings } \\
\hline LV ejection fraction (\%) & $34.1 \pm 3.5$ & $33.5 \pm 5.2$ & 0.76 \\
\hline$L V$ end-diastolic volume $\left(\mathrm{ml} / \mathrm{m}^{2}\right)$ & $106.0 \pm 93.0$ & $95.6 \pm 18.2$ & 0.14 \\
\hline LV end-systolic volume $\left(\mathrm{ml} / \mathrm{m}^{2}\right)$ & $69.7 \pm 6.3$ & $63.3 \pm 12.8$ & 0.2 \\
\hline LV end-diastolic pressure $(\mathrm{mmHg})$ & $12.2 \pm 6.7$ & $8.8 \pm 2.3$ & 0.12 \\
\hline Pulmonary wedge pressure $(\mathrm{mmHg})$ & $10.9 \pm 7.1$ & $7.6 \pm 2.0$ & 0.16 \\
\hline Mean pulmonary artery pressure $(\mathrm{mmHg})$ & $19.7 \pm 7.2$ & $15.0 \pm 3.1$ & 0.06 \\
\hline $\mathrm{RV}$ end-diastolic pressure $(\mathrm{mmHg})$ & $7.0 \pm 2.2$ & $7.4 \pm 1.8$ & 0.64 \\
\hline Mean right atrial pressure $(\mathrm{mmHg})$ & $5.8 \pm 2.6$ & $6.0 \pm 1.9$ & 0.82 \\
\hline Cardiac index $\left(L \cdot \mathrm{min}^{-1} \cdot \mathrm{m}^{-2}\right)$ & $2.1 \pm 0.6$ & $2.1 \pm 0.5$ & 0.83 \\
\hline \multicolumn{4}{|l|}{ Mitral regurgitation } \\
\hline Absent-mild & 9 & 12 & 0.99 \\
\hline Moderate-severe & 0 & 0 & \\
\hline \multicolumn{4}{|l|}{ Echocardiographic findings } \\
\hline LV end-diastolic dimension $(\mathrm{mm})$ & $58.7 \pm 3.7$ & $59.3 \pm 6.5$ & 0.81 \\
\hline LV end-systolic dimension ( $\mathrm{mm}$ ) & $49.0 \pm 5.5$ & $49.1 \pm 7.0$ & 0.91 \\
\hline Fractional shortening $(\%)$ & $16.6 \pm 6.7$ & $17.0 \pm 4.5$ & 0.86 \\
\hline Left atrial dimension ( $\mathrm{mm}$ ) & $39.2 \pm 5.3$ & $37.7 \pm 5.0$ & 0.5 \\
\hline \multicolumn{4}{|l|}{ Medication } \\
\hline Digitalis (\%) & 100 & 58 & 0.04 \\
\hline Diuretics (\%) & 100 & 92 & 0.99 \\
\hline ACE inhibitor (\%) & 67 & 83 & 0.61 \\
\hline$\beta$-blocker (\%) & 0 & 42 & 0.04 \\
\hline
\end{tabular}

MDCM, mildly dilated cardiomyopathy; NYHA, New York Heart Association; LV, left ventricular; RV, right ventricular; ACE, angiotensin converting enzyme.

Table 2 Changes in the Echocardiographic Findings

\begin{tabular}{lcccccccc}
\hline \hline & \multicolumn{3}{c}{ Patients without cardiac events } & & \multicolumn{2}{c}{ Patients with cardiac events } \\
\cline { 2 - 3 } & $\begin{array}{c}\text { Initial } \\
(n=11)\end{array}$ & $\begin{array}{c}\text { Follow-up } \\
(n=10)\end{array}$ & p value & & $\begin{array}{c}\text { Initial } \\
(n=9)\end{array}$ & $\begin{array}{c}\text { Follow-up } \\
(n=7)\end{array}$ & $p$ value \\
\hline LV end-diastolic dimension (mm) & $58 \pm 6$ & $50 \pm 8$ & $<0.001$ & & $59 \pm 4$ & $61 \pm 5$ & $N S$ \\
LV end-systolic dimension (mm) & $48 \pm 7$ & $38 \pm 11$ & $<0.001$ & & $49 \pm 6$ & $52 \pm 4$ & $N S$ \\
Fractional shortening (\%) & $17 \pm 5$ & $26 \pm 11$ & 0.007 & & $17 \pm 7$ & $14 \pm 3$ & $N S$ \\
Left atrial dimension (mm) & $38 \pm 5$ & $34 \pm 6$ & 0.03 & & $39 \pm 5$ & & $43 \pm 5$ & 0.02 \\
\hline
\end{tabular}

$L V$, left ventricular.

were used to compare 2 groups. A Cox proportional hazards regression model was used to identify independent factors predicting cardiac events. A p value less than 0.05 was considered statistically significant.

\section{Results}

Clinical Characteristics and Cardiac Events (Table 1)

During the follow-up period of $6.8 \pm 3.7$ years, there were 9 cardiac events ( 5 heart failure deaths, 2 sudden deaths, and 2 re-hospitalizations for heart failure). The dimensions and systolic function of the left ventricle were similar and there were no other differences in baseline characteristics between the 2 groups. Medical treatment did not differ significantly between the patients with and those without cardiac events, except for the use of $\beta$-blockers and digitalis: 5 of the 12 patients $(42 \%)$ without cardiac events received $\beta$-blockers compared with none of the patients with cardiac events.
Changes in the Echocardiographic Parameters (Table 2)

Of the 21 patients, follow-up echocardiographic data were available for 17 patients. The mean time to follow-up echocardiography was $5.5 \pm 3.4$ years. A significant decrease in the LV end-diastolic dimension and improvement in the fractional shortening were observed in patients without cardiac events. In contrast, there was no change in patients with cardiac events. Moreover, although the left atrial dimensions decreased slightly in patients without cardiac events, increased left atrial dimensions were observed at follow-up in patients with cardiac events.

\section{Proportional Hazard Analysis (Table 3)}

Based on a univariate proportional hazard model, LV end-diastolic pressure and mean pulmonary artery pressure at diagnosis, and the LV and left atrial dimensions at follow-up were significant predictors of poor outcome. Based on multivariate analysis, the left atrial dimensions were the only follow-up echocardiographic finding that was a significant predictor of poor outcome. 
Table 3 Predictors of Prognosis in Patients With MDCM

\begin{tabular}{|c|c|c|c|c|}
\hline & \multicolumn{2}{|c|}{ Univariate analysis } & \multicolumn{2}{|c|}{ Multivariate analysis } \\
\hline & Relative risk $(95 \% \mathrm{CI})$ & p value & Relative risk & $p$ value \\
\hline \multicolumn{5}{|l|}{ Clinical characteristics } \\
\hline Age at diagnosis & $0.93(0.86-1.01)$ & 0.98 & & \\
\hline Atrial fibrillation & $0.93(0.25-3.52)$ & 0.91 & & \\
\hline \multicolumn{5}{|l|}{ Hemodynamic findings } \\
\hline LV ejection fraction & $1.05(0.91-1.20)$ & 0.53 & & \\
\hline LV end-diastolic volume & $1.04(0.98-1.10)$ & 0.24 & & \\
\hline LV end-systolic volume & $1.03(0.96-1.10)$ & 0.42 & & \\
\hline LV end-diastolic pressure & $1.15(1.01-1.33)$ & 0.04 & & \\
\hline Pulmonary wedge pressure & $1.13(0.99-1.29)$ & 0.06 & & \\
\hline Mean pulmonary artery pressure & $1.15(1.01-1.29)$ & 0.02 & & \\
\hline RV end-diastolic pressure & $0.89(0.61-1.30)$ & 0.55 & & \\
\hline Mean right atrial pressure & $0.92(0.64-1.3)$ & 0.63 & & \\
\hline Cardiac index & $1.93(0.41-9.01)$ & 0.40 & & \\
\hline \multicolumn{5}{|c|}{ Echocardiographic findings at diagnosis } \\
\hline LV end-diastolic dimension & $0.97(0.86-1.10)$ & 0.65 & & \\
\hline LV end-systolic dimension & $0.98(0.88-1.08)$ & 0.66 & & \\
\hline Fractional shortening & $1.01(0.88-1.17)$ & 0.85 & & \\
\hline Left atrial dimension & $1.09(0.94-1.26)$ & 0.24 & & \\
\hline \multicolumn{5}{|l|}{ Echocardiographic findings at follow-up } \\
\hline LV end-diastolic dimension & $1.16(1.02-1.31)$ & 0.02 & & \\
\hline LV end-systolic dimension & $1.12(1.01-1.25)$ & 0.03 & & \\
\hline Fractional shortening & $0.89(0.79-1.02)$ & 0.09 & & \\
\hline Left atrial dimension & $1.28(1.08-1.52)$ & 0.005 & & \\
\hline LV end-diastolic dimension & & & 0.857 & 0.43 \\
\hline LV end-systolic dimension & & & 1.189 & 0.26 \\
\hline Left atrial dimension & & & 1.293 & 0.04 \\
\hline
\end{tabular}

MDCM, mildly dilated cardiomyopathy; $C I$, confidence interval; $L V$, left ventricular; RV, right ventricular.

\section{Discussion}

We have shown that the long-term prognosis of patients with MDCM is relatively poor, especially in patients with abnormal hemodynamic findings and progressive left atrial dilation.

\section{Previous Studies of Ventricular Dilatation and \\ Prognosis in Patients With DCM}

In earlier reports, the natural history of patients with DCM was for a poor prognosis, with a mortality rate of $50 \%$ at 5 years ${ }^{4,11}$ and despite advances in medical therapy, including angiotensin-converting enzyme inhibitors (ACEI) and $\beta$-blockers, the prognosis is still relatively poor, with a 5-year mortality of $20 \% 2,12,13$ The degree of LV end-diastolic dilatation is considered to be one of the primary prognostic predictors ${ }^{2-5}$ but Keren et al reported in 1985 that a subgroup of patients with DCM presented in end-stage heart failure despite mild LV dilatation6 Gavazzi et al studied 144 patients with DCM to determine the variability and prognostic importance of LV dilatation and reported that mild dilatation was not uncommon $(31 \%$ of patients with DCM)? These patients are a heterogeneous group that includes some in the early stages of the disease and others with severe LV dysfunction and minimal dilatation. However, those earlier reports have several limitations, such as the relatively short follow-up period (from 20 to 30 months) and the infrequent use of ACEI and $\beta$-blockers.

Ventricular Dilatation and Prognosis in the Present Study

The prevalence of MDCM was 15\% in our patient cohort, which is less than that reported by Gavazzi et al ${ }^{8}$ possibly because of differences in patient selection, criteria and the definition of MDCM. During the follow-up period, there were 9 cardiac events in the 21 patients with MDCM, and patients with impaired hemodynamics had an especially poor prognosis.

\section{Changes in the Echocardiographic Findings of \\ Patients With MDCM}

In a report with a follow-up period of 30 months, $19 \%$ of patients with MDCM had an increase in the size of the left ventricle and $16 \%$ had a decrease, but the changes in LV size and systolic function over a longer period have not been determined. In the present study, patients with cardiac events showed no change in LV size or systolic function, but did develop dilation of the left atrium during the follow-up period of approximately 7 years. In contrast, during that same time period patients without cardiac events showed a decrease in LV size and improvement in systolic function. Although the precise mechanisms of these results are unclear, there are several possibilities. First, spontaneous improvement in systolic function can occur in patients with DCM!4-17 Steimle et al reported that $27 \%$ of patients with recent onset DCM showed improvement in systolic function ${ }^{16}$ and they also noticed that the LV dimensions of patients who showed improvement were less than those of patients who did not. Therefore, there is a subset of patients with MDCM who will experience spontaneous improvement in systolic function, although, in our study, patients with alcohol-induced myocardial damage or acute myocarditis were carefully excluded. Second, none of the patients with cardiac events received $\beta$-blockers compared with $42 \%$ of the patients without cardiac events. However, it is also important to note that there was no significant difference in the initial hemodynamic findings between those who did and those who did not receive $\beta$-blockers. Although, to our knowledge, it has not been 
reported whether $\beta$-blockers should be given to patients with MDCM, it is well established that $\beta$-blockers can potentially reverse the remodeling of the left ventricle in patients with DCM or ischemic cardiomyopathy! 18,19 It is therefore possible that this treatment may have contributed to the decrease in LV size and improvement in systolic function observed in some patients without cardiac events. Finally, left atrial dimensions and function have been found to correlate with exercise capacity and prognosis in patients with DCM ${ }^{20-22}$ Modena et al reported that a dilated left atrium $(\geq 45 \mathrm{~mm})$ was an independent predictor of prognosis in 123 patients with $\mathrm{DCM}^{22}$ In our present study, there was no difference in the prevalence of atrial fibrillation and the severity of mitral regurgitation between the 2 groups and so left atrial dilation may have resulted from overall hemodynamic deterioration in the patients with cardiac events, although the precise mechanism remains undetermined.

In conclusion, patients with MDCM represent a common subgroup of DCM and the condition has 2 important features: one group of patients with early and mild DCM may stabilize or improve through the natural course or with appropriate medications; the other group shows definite impairment of hemodynamics together with left atrial dilation, despite modest LV dilation and it is this group which has the poorer prognosis and must be carefully followed.

\section{Study Limitations}

First, the study was retrospective with a small number of patients. Second, none of the patients with cardiac events were given $\beta$-blockers, because of the retrospective study design. Third, we did not quantify LV diastolic function. Finally, myocardial biopsies were not performed. Despite these limitations, we believe that the results from long follow-up period give an indication of the true prognosis of patients with MDCM.

\section{References}

1. WHO/ISFC task force on the definition and classification of cardiomyopathies. Circulation 1996; 93: 841-842.

2. Sugrue DD, Rodeheffer RJ, Codd MB, Ballard DJ, Fuster V, Gersh BJ. The clinical course of idiopathic dilated cardiomyopathy: A population-based study. Ann Intern Med 1992; 117: 117-123.

3. Doi YL, Chikamori T, Takata J, Yonezawa Y, Poloniecki JD, Ozawa T, et al. Prognostic value of thallium-201 perfusion defects in idiopathic dilated cardiomyopathy. Am J Cardiol 1991; 67: 188-193.

4. Diaz AR, Obasohan A, Oakley CM. Prediction of outcome in dilated cardiomyopathy. Br Heart J 1987; 58: 393-399.

5. Ikram H, Williamson HG, Won M, Grozier IG, Wells EJ. The course of idiopathic dilated cardiomyopathy in New Zealand. Br Heart $J$ 1987; 57: 521-527.

6. Keren A, Billingham ME, Weintraub D, Stinson E, Popp RL. Mildly dilated cardiomyopathy. Circulation 1985; 72: 302-309.

7. Keren A, Gottlieb S, Tzivoni D, Stern S, Yarom R, Billingham ME, et al. Mildly dilated congestive cardiomyopathy. Circulation 1990; 81: 506-517.

8. Gavazzi A, Maria RD, Renosto G, Moro A, Borgia M, Caroli A, et al. The spectrum of left ventricular size in dilated cardiomyopathy: Clinical correlates and prognostic implications. Am Heart J 1993; 125: $410-422$

9. Goodwin JF. The frontiers of cardiomyopathy. Br Heart J 1982; 48: $1-18$.

10. Schiller NB, Shah PM, Crawford M. Recommendations for quantification of the left ventricle by two-dimensional echocardiography. $J$ Am Soc Echocardiogr 1986; 2: 358-367.

11. Fuster V, Gersh BJ, Giuliani ER, Tajik AJ, Brandernburg RO, Frye RL. The natural history of idiopathic dilated cardiomyopathy. Am J Cardiol 1981; 47: 525-531.

12. Komajda M, Jais JP, Reeves F, Goldfarb B, Bouhour JB, Juillieres $\mathrm{Y}$, et al. Factors predicting mortality in idiopathic dilated cardiomyopathy. Eur Heart J 1990; 11: 824-831.

13. Azuma A, Matsuo A, Nakamura T, Kawasaki T, Yamamoto K, Hyogo M, et al. Improved survival of idiopathic dilated cardiomyopathy in the 1990s. Jpn Circ J 1999; 63: 333-338.

14. Figula HR, Rahlf G, Nieger M, Luig H, Kreuzer H. Spontaneous hemodynamic improvement or stabilization and associated biopsy findings in patients with congestive cardiomyopathy. Circulation 1985; 71: $1095-1104$.

15. Cross AM Jr, Steenbergen C, Higginbotham MB. Recovery of left ventricular function in acute nonischemic congestive cardiomyopathy. Am Heart J 1995; 129: 24-30.

16. Steimle AE, Stevenson LW, Fonarow GC, Hamilton MA. Prediction of improvement in recent onset cardiomyopathy after referral for heart transplantation. J Am Coll Cardiol 1994; 23: 553-559.

17. Kitaoka H, Takata J, Yabe T, Hitomi N, Furuno T, Doi YL. Low dose dobutamine stress echocardiography predicts the improvement of left ventricular systolic function in dilated cardiomyopathy. Heart 1999; 81: 523-527.

18. Waagstein F, Bristow MR, Swedberg K, Camerini F, Fowler MB, Silver MA, et al. Beneficial effects of metoprolol in idiopathic dilated cardiomyopathy. Lancet 1993; 342: 1441-1446.

19. Inoue S, Yokota Y, Takaoka H, Kawai H, Yokoyama M. Effect of beta-blocker therapy on ventricular arrhythmias in patients with idiopathic dilated cardiomyopathy. Jpn Circ J 2000; 64: 87-92.

20. Ito T, Suwa M, Kobashi A, Yagi H, Otake Y, Hirota Y. Prognostic value of left atrial appendage function in patients with dilated cardiomyopathy. Jpn Circ J 2000; 64: 340-344.

21. Triposkiadis F, Trikas A, Pitsavos C, Papadopoulos P, Toutouzas P. Relation of exercise capacity in dilated cardiomyopathy to left atrial size and systolic function. Am J Cardiol 1992; 70: 825-827.

22. Modena MG, Muia N, Sgura FA, Molinari R, Castella A, Rossi R. Left atrial size is the major predictor of cardiac death and overall clinical outcome in patients with dilated cardiomyopathy: A longterm follow-up study. Clin Cardiol 1997; 20: 553-560. 